\title{
Virtual integration of laboratories over long distance for real-time co-simulation of power systems
}

\author{
Marija Stevic, Steffen Vogel, Markus Grigull and \\ Antonello Monti \\ ACS, E.ON Energy Research Center \\ RWTH University \\ Aachen, Germany
}

\begin{abstract}
The interest in the virtual integration of hardware and software assets located at geographically dispersed locations, although not new, has spiked recently. However, realizing joint real-time simulation in connected laboratories is posing new challenges. This paper discusses the generalized requirements of a framework for the virtual integration of laboratories and presents the architecture of the platform that integrates two real-time digital simulators (RTDS located at ACS, RWTH Aachen University, Germany, and OPAL-RT at Politecnico di Torino, Italy). The platform enables remote and online monitoring of the entire interconnected system which is a step towards developing Simulation as a Service concept. The application of this platform for real-time co-simulation of interconnected transmission and distribution systems is demonstrated.
\end{abstract}

Index Terms-Distributed simulation, real-time simulation, transmission systems, distribution systems

\section{INTRODUCTION}

Geographically distributed real-time simulation framework for power system analysis and testing is an advanced concept that extends the capabilities of one realtime simulator and (Power) Hardware-in-the-Loop testing. The concept enables the integration of laboratories with different hardware assets located far from one another. This framework for virtual integration of laboratories is of great interest in particular for studies on future energy systems, since the current energy transition calls for transformation of system design, planning and testing tools and methods. Interdisciplinary and more integrated system level studies are required to evaluate the synergies of new components and concepts in future energy systems and to identify potential unpredictable interactions [1]. To this aim, the Energy Union strategy proposed by the European Commission [2] emphasizes the importance of fully coordinated and integrated approach to research and innovation for a more secure, affordable and sustainable energy system.

Virtual integration of laboratories allows for expanding capabilities of individual laboratories to address the requirements for studies of large-scale, system level and interdisciplinary scenarios. Moreover, it provides a flexible

\author{
Abouzar Estebsari, Enrico Pons, Tao Huang and \\ Ettore Bompard \\ Department of Energy \\ Politecnico di Torino \\ Turin, Italy
}

virtual environment for technical cooperation, challenged and often impaired by confidentiality aspects [3]. This is typically the case in scenarios that refer to interoperability studies of components and devices from different vendors or between operators. Confidentiality issues are often the main obstacle to perform detailed simulation and in-depth testing before actual operation in a real system.

The idea of virtual integration of hardware and software assets located at geographically dispersed locations has been investigated already for a while [4] [5]. In recent years there has been a significant increase in interest within the simulation community in the concept of interconnecting laboratories over the Internet. Remote execution of experiments was investigated in [6]. Feasibility, applicability and benefits of a real-time virtual connection of laboratories over Internet for joint operation in a single experiment were demonstrated for virtual integration of simulators [7][8] and hardware assets [9]. This paper gives an insight into requirements for a framework for real-time virtual integration of laboratories over long distance going beyond basic prerequisites for the infrastructure operation and including utilization of the infrastructure from third parties. Furthermore, an Internet-distributed simulation platform that integrates in real time laboratories at ACS (RWTH Aachen University, Germany) and PoliTo (Politecnico di Torino, Italy) is introduced. Implementation of a remote and online monitoring of the simulation execution via a web browser is briefly described. Finally, the platform is applied to real-time co-simulation of transmission and distribution systems that are based on parameters derived from steady state snapshots of real networks.

\section{REQUIREMENTS OF A FRAMEWORK ENABLING VIRTUAL INTEGRATION OF LABORATORIES}

A framework for virtual integration of laboratories should be designed with the goal of enabling flexible integration of the resources available at each laboratory, and, at the same time, a flexible utilization of an infrastructure as a whole. In this respect, an essential requirement for such framework is to provide a specific set of interfaces and services. Namely, hard real-time interfaces are necessary for the integration of various hardware and software assets at laboratories, to

This work received financial support from the KPN project "ProOfGrids (ref. n. 215942/E20)" financed by the Research Council of Norway's RENERGI program and industry partners (EDF, NationalGrid, Siemens, Statkraft, Statnett, Statoil). 
enable their joint operation in a single experiment. Moreover, high-level interfaces, such as user interface and interface for data logging, are required to enable the interaction with an experiment and post-processing of results for further analyses. Additionally, high-level services, such as Simulation as a Service and Data as a Service, are required to provide flexible access to third parties and leverage utilization of the infrastructure.

The development of the described interfaces and services imposes many diverse and challenging requirements to be met within a framework for virtual integration of laboratories. A basic example of a conceptual architecture diagram for such framework is illustrated in Figure 1.

As it is depicted in Figure 1, the framework should allow for multi-terminal virtual integration of laboratories. Each laboratory may offer for usage multiple hardware and software assets that are of different kinds such as real-time digital simulators [10] (i.e. RTDS, OPAL-RT), measurement devices (i.e. Phasor Measurement Unit), estimation and control algorithms to name just a few. Laboratory-toLaboratory (Lab2Lab) interface refers to an interface necessary for integration of different types of hardware and software assets located at multiple laboratories into a single experiment.

Fast and reliable communication between Lab2Lab interfaces is a basic requirement for real-time co-simulation and integration of Hardware-in-the-Loop setups. This requirement represents the main obstacle in applying the concept of virtual integration of geographically dispersed assets in case of long distance between the laboratories. It is not realistic to assume availability of a dedicated communication infrastructure in all cases and it is difficult to guarantee hard real-time behavior of a communication link over a shared communication medium such as Internet. It is important to emphasize that even if hard real-time communication link is assumed, the impact of the communication delay on experiment execution must be compensated by means of interface algorithms to ensure fidelity of results.

Cloud platform refers to a platform that hosts services which are available on demand. These services support remote access and user interaction with the infrastructure, post-processing of data and other high-level services. Laboratory-to-Cloud (Lab2Cloud) interface refers to an interface required at each laboratory as an interface to the described services. Main difference between Lab2Lab and Lab2Cloud interfaces is the requirement for hard real-time integration that is not a prerequisite for Lab2Cloud interface. Data exchange between Lab2Cloud interface and the cloud platform is required in soft real-time manner in case online interaction with the experiment is required. Otherwise, data exchange in non-real-time is satisfactory. An example of such case is data collection for a historical database to enable postprocessing and post-analysis of the results of an experiment.

Flexibility and scalability in terms of enabling integration of new laboratory facilities and hardware and software assets should be planned in early stage of the framework development. Another important aspect for rapid development is portability of Lab2Lab and Lab2Cloud interfaces among laboratories and for integration of different local assets. Access to the platform and cloud-based services should be processed via a secure identity management system. With respect to data security of data exchange among laboratories, a virtual private network can be considered as a solution. Ensuring security of assets at each laboratory, in particular of hardware assets, is of high priority in the context of connecting laboratories over a communication medium.

\section{INTERNET-DISTRIBUTED SIMULATION PLATFORM AND EXPERIMENT}

Following the overall vision and requirements for a framework for virtual integration of laboratories, an Internet-

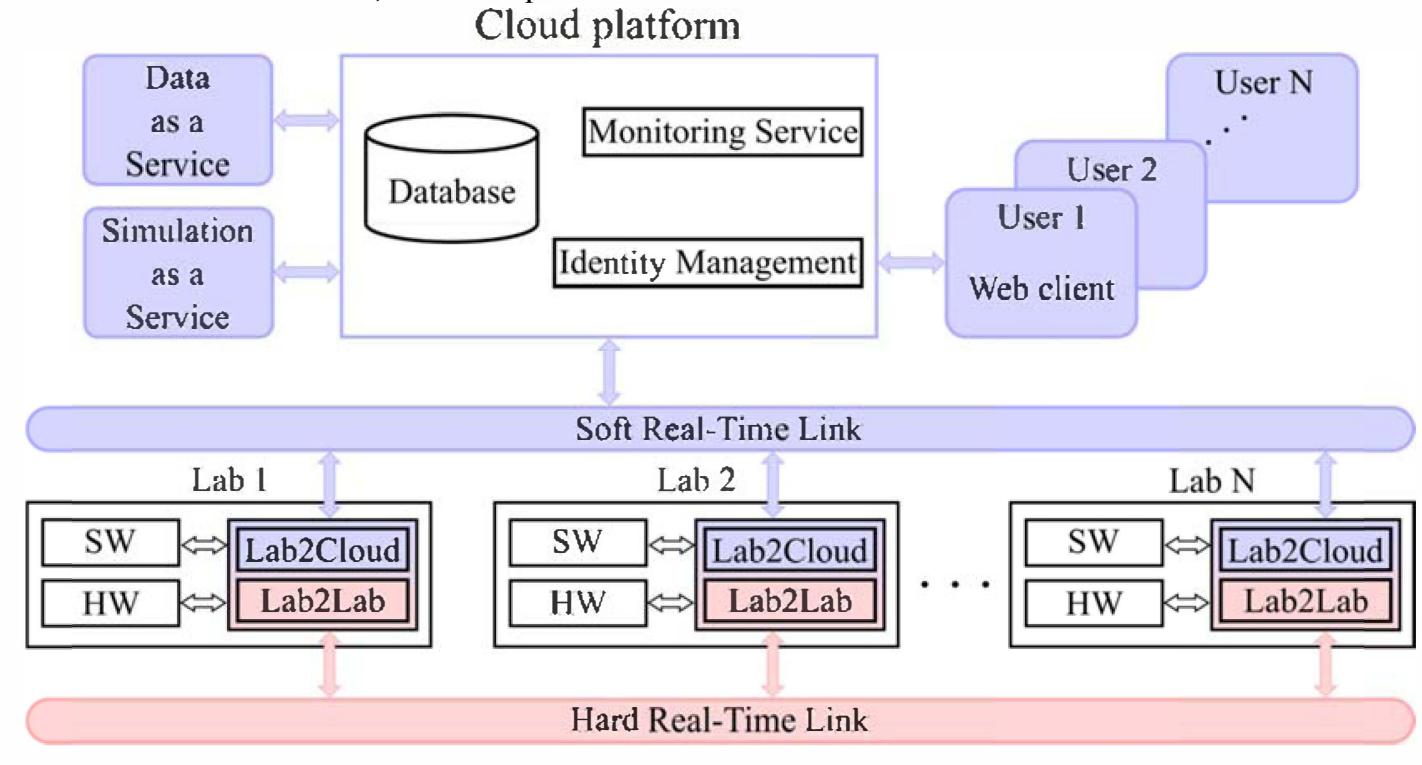

Figure 1. Architecture diagram of a framework for virtual integration of laboratories 
distributed simulation (InetDistSim) platform that integrates in real time laboratories across wide area network was developed and introduced in [7]. Feasibility of integration of two real-time digital simulators, OPAL-RT (located at SINTEF, Norway) and OPAL-RT (located at ACS, RWTH Aachen University, Germany), in real-time co-simulation was demonstrated. Applicability of the platform for simulation of high-voltage dc point-to-point link which connects two ac systems was presented. This work further exploits the InetDistSim platform by integrating a laboratory at another location for application of a different simulation use case. Furthermore, the platform is extended to provide a web-based remote monitoring of the co-simulation results.

\section{A. Internet-distributed simulation platform - InetDistSim}

In this work, the InetDistSim platform is utilized for connecting laboratories at ACS (RWTH Aachen University, Germany) and PoliTo (Politecnico di Torino, Italy). More precisely, two real-time digital simulators, RTDS located at ACS and OPAL-RT located at PoliTo are integrated for cosimulation in real time. The high-level architecture of the InetDistSim platform is illustrated in Figure 2.

Design and development of the InetDistSim platform was driven by generalized requirements of architecture for virtual integration of laboratories descried in II. Simulator-tosimulator (Sim2Sim) server at each laboratory acts as a gateway between local simulators and the Internet. With respect to Figure 1, it provides both Lab2Lab and Lab2Cloud interfaces. Sim2Sim server is in-house developed software that runs real-time-patched version of Linux [11] with a minimal Fedora installation to reduce impact of background tasks on data exchange between the simulators. Independent processes take care of Lab2Lab and Lab2Cloud interfaces. Furthermore, processes for sending and receiving data in terms of Lab2Lab interface are asynchronous and independent, thus the additional delay that might be caused by waiting for a response from a remote server is avoided.

Data exchange between ACS and PoliTo laboratories is performed over the Internet, more precisely over national research and education networks. Sim2Sim server forwards data between simulators and remote Sim2Sim instances, drops invalid packets, collects statistics of the communication link and runs arbitrary operations on the forwarded data if required. Sim2Sim servers exchange data in from of User Datagram Protocol (UDP) for the purpose of data exchange between the simulators. UDP is selected as a preferable option compared to the connection-oriented counterpart Transmission Control Protocol (TCP). In terms of real-time data exchange it is preferable to discard reordered and lost packets instead of waiting for a packet retransmission. To provide security a Virtual Private Network (VPN) is utilized. The VPN solution is based on the open source VPN software Tinc [12] which is running directly on the Sim2Sim servers. This VPN daemon establishes a completely decentralized and fully meshed virtual network on top of the Internet and enables point-to-point data exchange which is not typical for other VPN solutions.

With reference to previous work in [7], the InetDistSim platform is additionally extended to enable consolidated and online monitoring of the simulation execution through a web application. The platform extension for remote and flexible monitoring represents a step towards development of more general concepts such as Simulation as a Service and Data as a Service as described in II. For this purpose, a public server is included in the platform. A public server accommodates webserver [13] and FIWARE Publish/Subscribe Context Broker [14][15].

FIWARE Context Broker represents a mediator between context producers and context consumers, which are in this case laboratories and web applications, respectively Furthermore, a user interaction can be enabled in a similar manner where a web application acts as a context producer. As already mentioned, Sim2Sim server at each laboratory manages simulator interface to the Context Broker besides the interface for data exchange between laboratories, i.e. simulators. Sim 2 Sim server receives preselected set of simulation data from the local simulator, adds a timestamp and publishes data to the Context Broker with a predefined sending rate. The Context Broker makes the latest received simulation data from both Sim2Sim servers available on

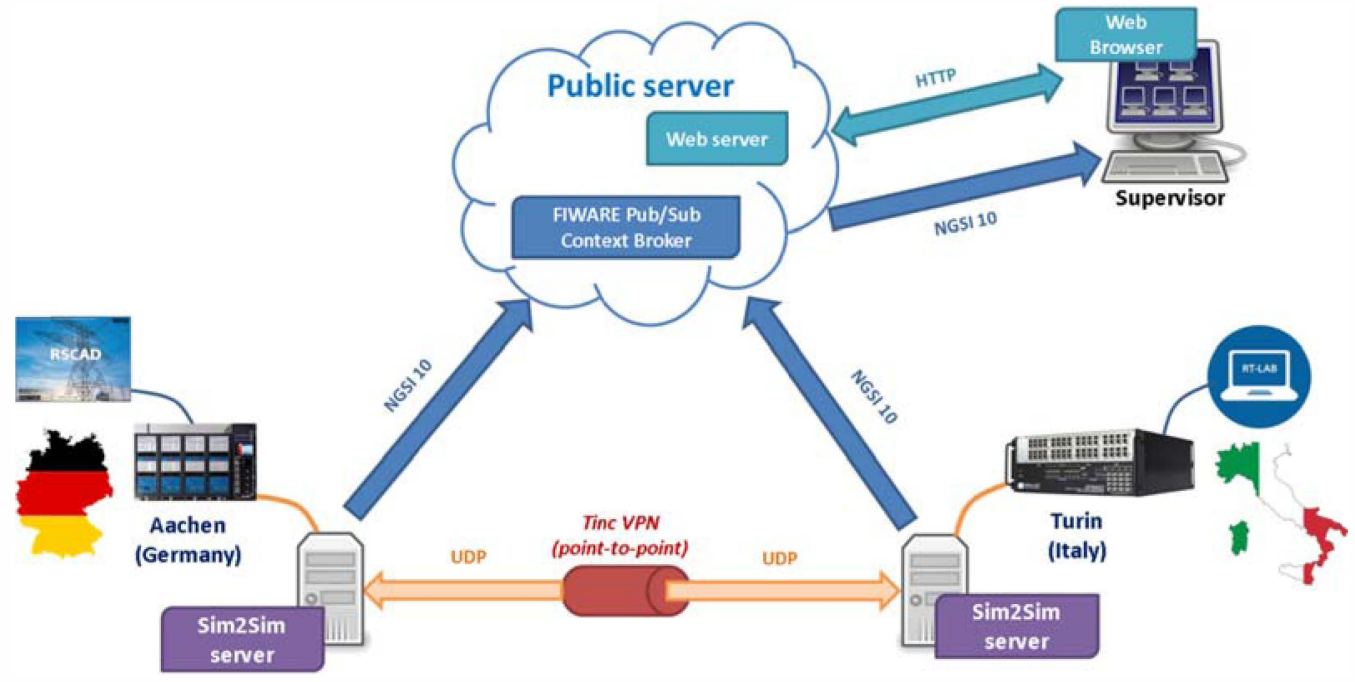

Figure 2. High-level architecture of the Internet-distributed simulation platform 
request to web applications. A user can access the consolidated overview of the simulation performed at both laboratories based on visualization service via a web browser.

\section{B. Internet-distributed simulation experiment: real-time co- simulation of transmission and distribution systems}

In this work, the platform is applied for real-time cosimulation of transmission and distribution systems. Namely, the RTDS system at ACS simulates the transmission grid whereas the OPAL-RT system at PoliTo simulates distribution grid. Realistic systems are adopted as a case study. The transmission system represents an area in Piedmont region, Italy. The system includes in total 41 buses of voltage levels $380 \mathrm{kV}$ and $220 \mathrm{kV}$. STURA substation $(380 / 220 \mathrm{kV})$ in Turin represents a terminal where a distribution system, which is simulated at another laboratory, is connected to the transmission system. Simulated distribution system refers to a portion of the distribution system in the city of Turin. The system consists of a primary substation with three $22 \mathrm{kV}$ busbars, each fed by a transformer characterized by voltage ratio of $220 / 22 \mathrm{kV}$. Number of MV feeders included in the system is five and the total number of MV buses is 49 .

An important aspect of the InetDistSim platform is a simulator-to-simulator interface between the two subsystems, in this case transmission and distribution systems, which are simulated at geographically dispersed locations. A hybrid approach to the design of a simulator-to-simulator interface, which was proposed in [7], is applied in this work for integration of transmission and distribution systems. In this approach each simulator performs simulation in time domain, whereas the interface quantities are represented in terms of their time-varying Fourier coefficients, known as dynamic phasors. The time-frequency domain interface was further analyzed in [8] where high simulation fidelity in case of steady state and slow transient was demonstrated. The interface delay is compensated by means of phase shift applied on the received dynamic phasor. The average round trip delay between PoliTo and ACS is in the range of $27 \mathrm{~ms}$. Described interface characteristics limit the interface bandwidth and applicability of the simulation framework to the studies of power system phenomena with time constants $>300 \mathrm{~ms}$. However, faster local transients can be studied as long as they are damped locally and do not propagate through the interface. Capabilities of the interface that were described satisfy requirements of studies in this work.

\section{Results of the Internet-distributed simulation experiment}

Real-time co-simulation of the interconnected transmission and distribution system allows for studies of how different levels of distributed generation, EV penetration and consumer behavior patterns in the distribution system, would affect the system operation at both transmission and distribution levels. It demonstrates the need of large-scale power system simulation consisting detailed simulation models of both transmission and distribution grids. If the model is too large to be simulated in a single local machine, it needs to be split and co-simulated in more than one machine (laboratory). It should be noted that what makes a system large is not only the size of the model in terms of components number, but also the simulation time step.

In this work, we assessed transmission and distribution system operation under extreme consumer/prosumer behavior at the distribution level. The integration of variable renewable energy is challenging in three different time scales: less than a few seconds, stability of the system faces challenges, from some minutes to some days, system demand-supply may loss balance, and in long term (some months or even years), the adequacy of the power system becomes more important to meet system peak demand. Our current analysis focuses on the balancing challenges from variable renewable energies, more specifically from PV generators. In a regular operation in analyzed distribution grid, there are four PV generators that contribute in supplying local consumers.

An extreme scenario is analyzed when the PV generation is assumed to drop (e.g. a big cloud appears suddenly and change solar irradiance due its intrinsic variability and uncertainty characteristic) at the same time when the residential consumers are increasing their consumption plugging EVs and utilizing more appliances, as illustrated in Figure 3. It results in a sudden increase in power absorption from the substation connected to the transmission system.

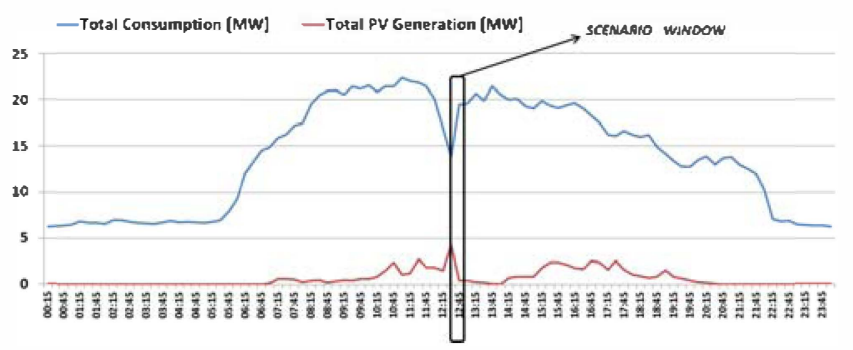

Figure 3. Indication of scenario window in 24-hour total load/generation profiles

Web application based monitoring provides visualization of online simulation data acquired from both transmission and distribution systems. This system overview is useful not only for third parties, but it extends simulation monitoring available at individual laboratories that is limited to the local system (i.e. transmission or distribution system). Furthermore, it is possible to limit consolidated monitoring to a specific set of data in case of confidentiality issues. The figures presented in the following section are obtained from the web application developed for overall system monitoring.

Figure 4 indicates voltage drop at the end of a feeder in distribution system caused by the described prosumer behavior. Web application offers a set of measurements that can be selected for monitoring.

Monitoring of transmission system simulation is shown in Figure 5. Frequency measurement at one of the neighboring buses to the substation where distribution system is connected is selected for monitoring. Although the rotating inertia of the large conventional generators mitigates fluctuations in the studied scenario, high penetration of PV generators could eventually have a significant impact on the transmission grid. 


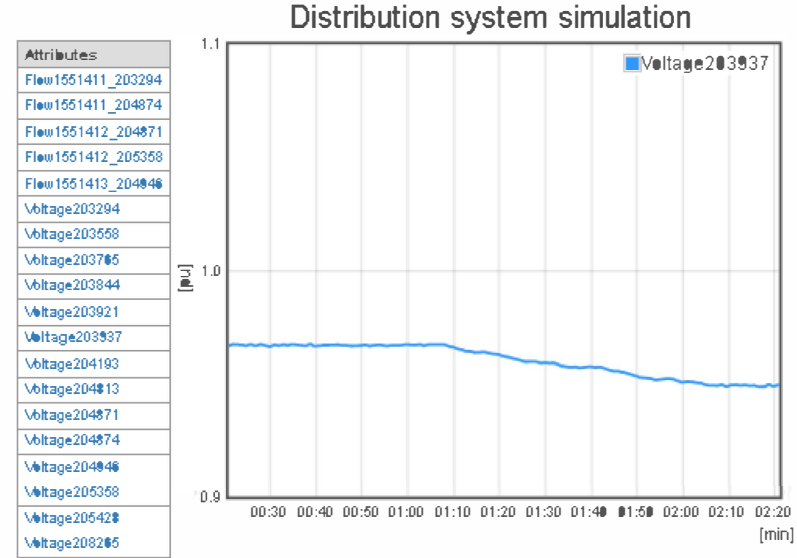

Figure 4. Monitoring of distribution system - RMS voltage at the end of the feeder

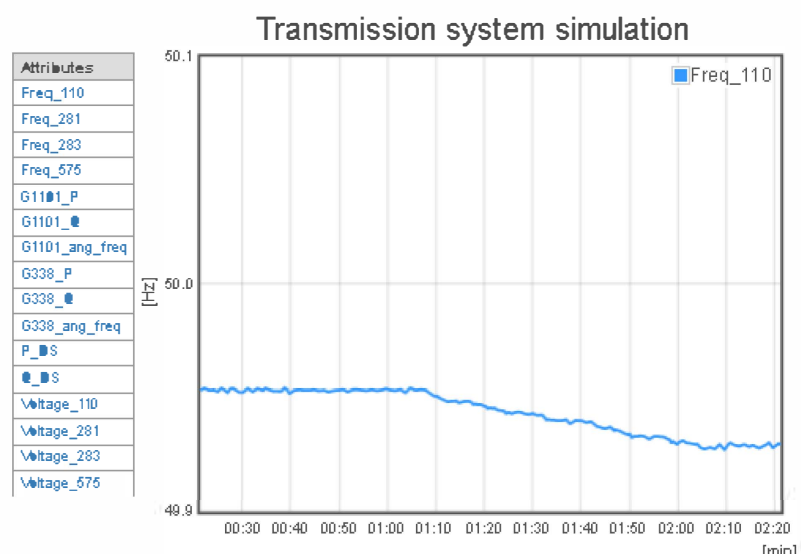

Figure 5. Monitoring of transmission system - frequency measurement

Obtained simulation results demonstrate the InetDistSim as a functional real-time co-simulation platform that can accommodate large-scale scenarios and provide a consolidated monitoring of the entire systems.

\section{CONCLUSION}

A novel concept of virtual integration of laboratories over long distance was investigated in this paper. A platform that integrates real-time digital simulators over Internet and enables online monitoring based on a web application was presented. Applicability of the platform and the overall concept was demonstrated for co-simulation of the transmission and distribution systems simulated at different locations. The concept of virtual integration of two real-time digital simulators over the Internet addresses the issue of the system size since the entire model requires large computational resources. Simulation in real time allows for future integration of hardware assets for Power Hardware-inthe-Loop testing such as PV inverters. Co-simulation of detailed transmission and distribution systems enables assessment of system level to component level aspects.

As a proof of concept, the platform was applied for assessing the effect of an extreme scenario in distribution system on both transmission and distribution system operation. Transmission and distribution system have been built from a steady state snapshot of real systems and represent a portion of Pidemont region and the city of Turin in Italy, respectively. Implementation of these models is meant to represent a realistic situation in which confidentiality aspects of sharing data and models among operators might limit system level and wide area studies required to evaluate interoperability and identify possible unacceptable interactions.

\section{ACKNOWLEDGMENT}

The authors gratefully acknowledge the support provided by the Institute for Energy and Transportation within European Commission Joint Research Centre (IET-JRC).

\section{REFERENCES}

[1] Beerten, J.; D Arco, S.; Suul, J.A., "Identification and Small-Signal Analysis of Interaction Modes in VSC MTDC Systems," in Power Delivery, IEEE Transactions on, vol.PP, no.99, pp.1-1

[2] A Framework Strategy for a Resilient Energy Union with a Forward Looking Climate Change Policy, European Commission

[3] Verseille, J.; Staschus, K., "The Mesh-Up: ENTSO-E and European TSO Cooperation in Operations, Planning, and R\&D," in Power and Energy Magazine, IEEE, vol.13, no.1, pp.20-29, Jan.-Feb. 2015

[4] J. L. Bastos, J. Wu, N. Schulz, R. Liu, and A. Monti, "Distributed simulation using the Virtual Test Bed and its real-time extension," in Proceedings of the Summer Computer Simulation Conference (SCSC '07), pp. 757-765, July 2007

[5] Faruque, M.O.O.; Sloderbeck, M.; Steurer, M.; Dinavahi, V., "Thermoelectric co-simulation on geographically distributed real-time simulators," in Power \& Energy Society General Meeting, 2009. PES '09. IEEE, vol., no., pp.1-7, 26-30 July 2009

[6] Weimer, J.; Yuzhe Xu; Fischione, C.; Johansson, K.H.; Ljungberg, P.; Donovan, C.; Sutor, A.; Fahlen, L.E., "A Virtual Laboratory for MicroGrid information and communication infrastructures," in Innovative Smart Grid Technologies (ISGT Europe), 2012 3rd IEEE PES International Conference and Exhibition on , vol., no., pp.1-6, 14-17 Oct. 2012

[7] Marija Stevic, Steffen Vogel, Antonello Monti, Salvatore D'Arco, "Feasibility of geographically distributed real-time simulation of HVDC system interconnected with AC networks," in PowerTech, 2015 IEEE Eindhoven, June 2015

[8] Stevic, M.; Benigni, A.; Monti, A.; "Development of a simulator-tosimulator interface for geographically distributed simulation of power systems in real time," in Industrial Electronics Society, IECON 2015 41st Annual Conference of the IEEE

[9] Palmintier, B.; Lundstrom, B.; Chakraborty, S.; Williams, T.; Schneider, K.; Chassin, D., "A Power Hardware-in-the-Loop Platform With Remote Distribution Circuit Cosimulation," in Industrial Electronics, IEEE Transactions on , vol.62, no.4, pp.2236-2245, April 2015

[10] Real-Time Simulation Technologies for Power Systems Design, Testing, and Analysis," in Power and Energy Technology Systems Journal, IEEE , vol.2, no.2, pp.63-73, June 2015

[11] Linux RT-patch [Online]. Available: https://rt.wiki.kernel.org/.

[12] Tinc VPN Project Home Page. [Online]. Available: http://tinc-vpn.org. [Accessed: 08.11.2015]

[13] Nginx Home Page. [Online]. Available: http://nginx.org. [Accessed: 08.11.2015]

[14] Fiware project Home Page. [Online]. Available: https://www.fiware.org/. [Accessed: 08.11.2015]

[15] Fiware project catalogue - Publish/Subscribe Context Broker. [Online]. Available:

http://catalogue.fiware.org/enablers/publishsubscribe-context-brokerorion-context-broker. [Accessed: 08.11.2015] 